\title{
Pharmacological treatment of acute spinal cord injury: current status and future prospects
}

\author{
M B Bracken PhD MPH \\ Professor and Vice Chairman, Department of Epidemiology and Public Health, Yale \\ University School of Medicine, 60 College Street, PO Box 3333, New Haven, Connecticut \\ 06510, USA.
}

The history and results of the National Acute Spinal Cord Injury Studies (NASCIS) are briefly reviewed. The current status of pharmacological therapy for acute spinal cord injury and future prospects are also summarized.

Key words: spinal cord injury; pharmacological treatment.

The possibility of pharmacologically treating acute spinal cord injury, particularly the post injury destruction of neurons by oxygen-free radical induced lipid peroxidation, has been suspected for many years. ${ }^{1}$ Several pharmacological agents have been examined in a variety of animal models but with conflicting results. Differences in animal models, injury models, drug dosing protocols, methods for evaluating neurological recovery, together with the small number of animals usually studied, prevent unequivocal conclusions being drawn from most of this literature.

Methylprednisolone is a notable exception, being investigated in one of the most scientifically valid series of animal experiments, starting with low doses in the order of $1.0 \mathrm{mg} / \mathrm{kg}$, and then later doses of 15 and $30 \mathrm{mg} / \mathrm{kg}$. This work has recently been reviewed by Hall. ${ }^{2}$

In 1977 the National Acute Spinal Cord Injury Study (NASCIS) was established to empirically investigate, in multicenter randomized controlled trials, the safety and efficacy of pharmacological agents of potential value in the early treatment of acute spinal cord injury. After a 2 year feasibility study to ensure that different medical centers could follow common study protocols the first study (NASCIS 1) was launched. NASCIS 1 compared 2 doses of methylprednisolone: the low dose consisted of a 15 minute bolus of $0.1 \mathrm{gm}$ followed by 10 daily doses of $0.1 \mathrm{~g}$ administered by i.v. push
( $25 \mathrm{mg}$ every 6 hours). The high dose regimen was exactly the same except for a tenfold increase in the dose. A total of 330 patients were randomized to the trial; however, no significant difference in neurological recovery at 6 weeks or 6 months $^{3}$ or one year ${ }^{4}$ after injury was observed.

In 1985, NASCIS 2 was started. Whereas the earlier trial had not included a placebo group, because of the existing widespread use of (very low doses of) steroids and for fear of litigation by some of the participating surgeons, the results of NASCIS 1 provided a rationale for subsequently using a placebo comparison group. In view of the promising animal work with the $30 \mathrm{mg} / \mathrm{kg}$ dose of methylprednisolone ${ }^{5}$ this dose became one of the treatment arms under study. A series of animal studies using naloxone ${ }^{6}$ led us to include this as a third treatment arm. The treatment arms for NASCIS 2 are summarized in Table 1.

In addition to the primary study hypotheses concerning drug efficacy and safety, the study hypothesized that patients treated relatively quickly after injury would be more likely to exhibit any therapeutic effects of drug therapy than those treated later. Further, it was postulated that any evidence of neurological effect on recovery would be seen primarily in those patients who were defined as being incomplete (ie with either some sensory or motor preservation below the level of lesion at the time of the initial examination). 
Table 1 NASCIS 2 study protocols

1 Naloxone: 15 minute bolus $5.4 \mathrm{mg} / \mathrm{kg}\left(200 \mathrm{mg} / \mathrm{m}^{2}\right) 23$ hour infusion $4.0 \mathrm{mg} / \mathrm{kg} / \mathrm{hr}$ $\left(150 \mathrm{mg} / \mathrm{m}^{2} / \mathrm{hr}\right)$

2 Methlyprednisolone: 15 minute bolus $30 \mathrm{mg} / \mathrm{kg}\left(1100 \mathrm{mg} / \mathrm{m}^{2}\right) 23$ hour infusion $5.4 \mathrm{mg} / \mathrm{kg} / \mathrm{hr}$ $\left(200 \mathrm{mg} / \mathrm{m}^{2} / \mathrm{hr}\right)$

3 Placebo: administered through both the 'naloxone' and 'methylprednisolone' pumps as 15 minute bolus and 23 hour infusion.

\section{Summary of NASCIS 2 results}

In May of 1990, the results of the 6 week and 6 month follow up examinations of the 487 people randomized in NASCIS 2 were published. ${ }^{7}$ The study demonstrated that patients treated with methylprednisolone, within 8 hours of injury, showed significantly greater neurological recovery than those treated with placebo. These results have recently been shown to essentially persist one year after injury. ${ }^{8}$ While naloxone-treated patients also generally showed greater neurological recovery than placebo, these differences could not be distinguished from chance in the analyses done to date in this study. Naloxone is not considered further in the present report.

A summary of the results of the methylprednisolone and placebo comparisons for NASCIS 2 patients, treated within 8 hours of injury, is presented in Figure 1. Here the neurological change score, reported in the earlier more detailed statistical analyses of these data $^{7,8}$, is reported as a function of the neurological deficit observed in the emergency room. It reflects what proportion of the initial neurological loss, observed in the emergency room, has been recovered at each follow up period. Thus:

Percent recovery $=$

Change in neurological

$\frac{\text { score at follow up }}{\text { Normal score }- \text { emergency }} \times 100$

room neurological score

Figure 1, upper panel, shows the relative recovery in motor function of the placebo and methylprednisolone-treated patients 6 weeks, 6 months and one year after injury. At 6 weeks, the placebo-treated patients recovered $11.6 \%$ of their lost neurological function compared with $18.6 \%$ of methylprednisolone-treated patients. At 6 months, placebo-treated patients had recovered $19.7 \%$ of their initially lost motor function compared with $28.6 \%$ in the methylprednisolone group. At one year, the respective recovery rates were $21.4 \%$ and $30.2 \%$. The asterisks reflect significance testing for these comparisons from the analyses of variance reported previously. ${ }^{7,8}$

The lower 2 panels of Figure 1 show the data for pinprick and light touch sensation. A similar pattern of results is observed to that seen for motor function, although fewer comparisons achieve nominal statistical significance reflecting generally greater variability in the sensory outcomes.

Figure 2 shows the recovery of patients grouped by whether they were neurologically complete or incomplete on their emergency room examination.

The upper panel of Figure 2 indicates that methylprednisolone-treated patients who had neurologically complete injuries in the emergency room (with no motor or sensory function below level of lesion) recover significantly more of their motor function if treated with methylprednisolone within 8 hours of injury compared with placebo. This enhanced recovery is also seen at 6 months and one year. As expected, overall recovery in the incomplete patients is considerably more than the complete patients, irrespective of treatment. The neurologically incomplete patients also recovered significantly more motor function if treated with methylprednisolone. Similar results are observed for recovery of pinprick and light touch sensation.

\section{Current status of pharmacological therapy}

There have been no empirical studies to 

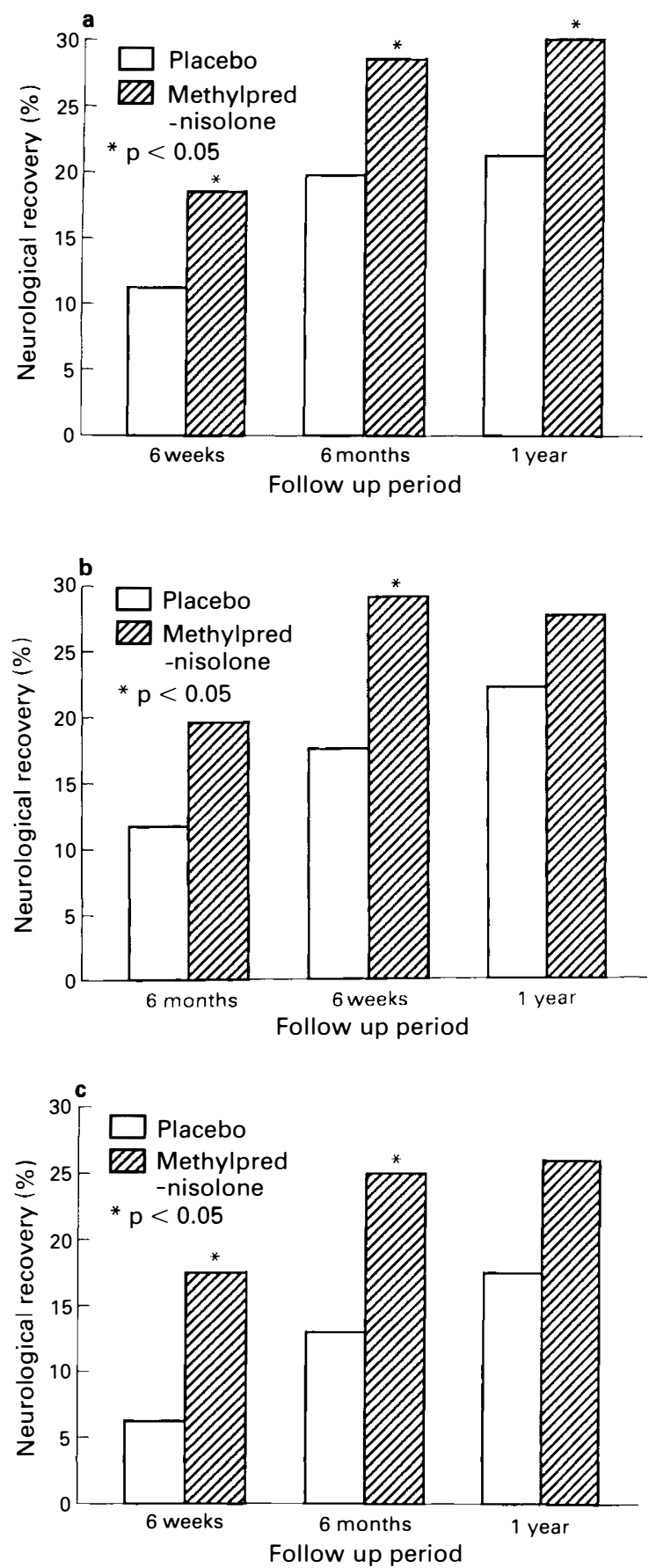

Figure 1 Percent recovery of (a) motor function, (b) pinprick and (c) light touch sensation 6 weeks, 6 months and one year after injury in patients treated with placebo or methylprednisolone within 8 hours of injury. $p$-values refer to analysis of variance ${ }^{7,8}$. indicate, at the present time, the frequency of using methylprednisolone to treat acute spinal cord injury. Anecdotal evidence suggests that the NASCIS 2 methylprednisolone regimen, including roadside administration of a bolus dose, is common practice in many parts of North America, Europe, Australia and Japan. The regulatory agencies of at least 8 countries have approved acute spinal cord injury as an indication for using methylprednisolone. There have also been anecdotal reports of high dose methylprednisolone being used prophylactically during surgical procedures where the spinal cord is at risk of iatrogenic injury.

There have been no other large, multicenter Phase 3 randomized trials of any other pharmacological agent (or, indeed, any other form of clinical management) used for treating acute spinal cord injury. Methylprednisolone remains the only form of management shown empirically in a Phase 3 trial to have efficacy in treating this injury. The NASCIS studies clearly indicate that methylprednisolone must be administered in the $30 \mathrm{mg} / \mathrm{k}$ range, following the NASCIS regimen and starting treatment within 8 hours of injury. Based on the pharmacodynamics of methylprednisolone, and the animal injury models, it is reasonable to postulate that the earlier after injury drug administration begins the greater the chance of benefit; however, there are no human data to address this.

There is no evidence that higher doses of methylprednisolone would be effective, and in cat studies, a $60 \mathrm{mg} / \mathrm{kg}$ dose proved ineffective. NASCIS 1 and other animal studies indicate that lower doses of methylprednisolone are unlikely to be effective. Nor can it be assumed that other steroids used for CNS trauma, will have the same neurological effects as methylprednisolone, particularly given the proposed mechanisms of lipid peroxidation inhibition thought to induce its effect. The importance of limiting treatment by methylprednisolone to patients whose treatment can begin within 8 hours of injury (which should be possible in virtually all patients) is emphasized by evidence in the NASCIS 2 data that patients given methylprednisolone have generally worse neurological outcomes than patients 

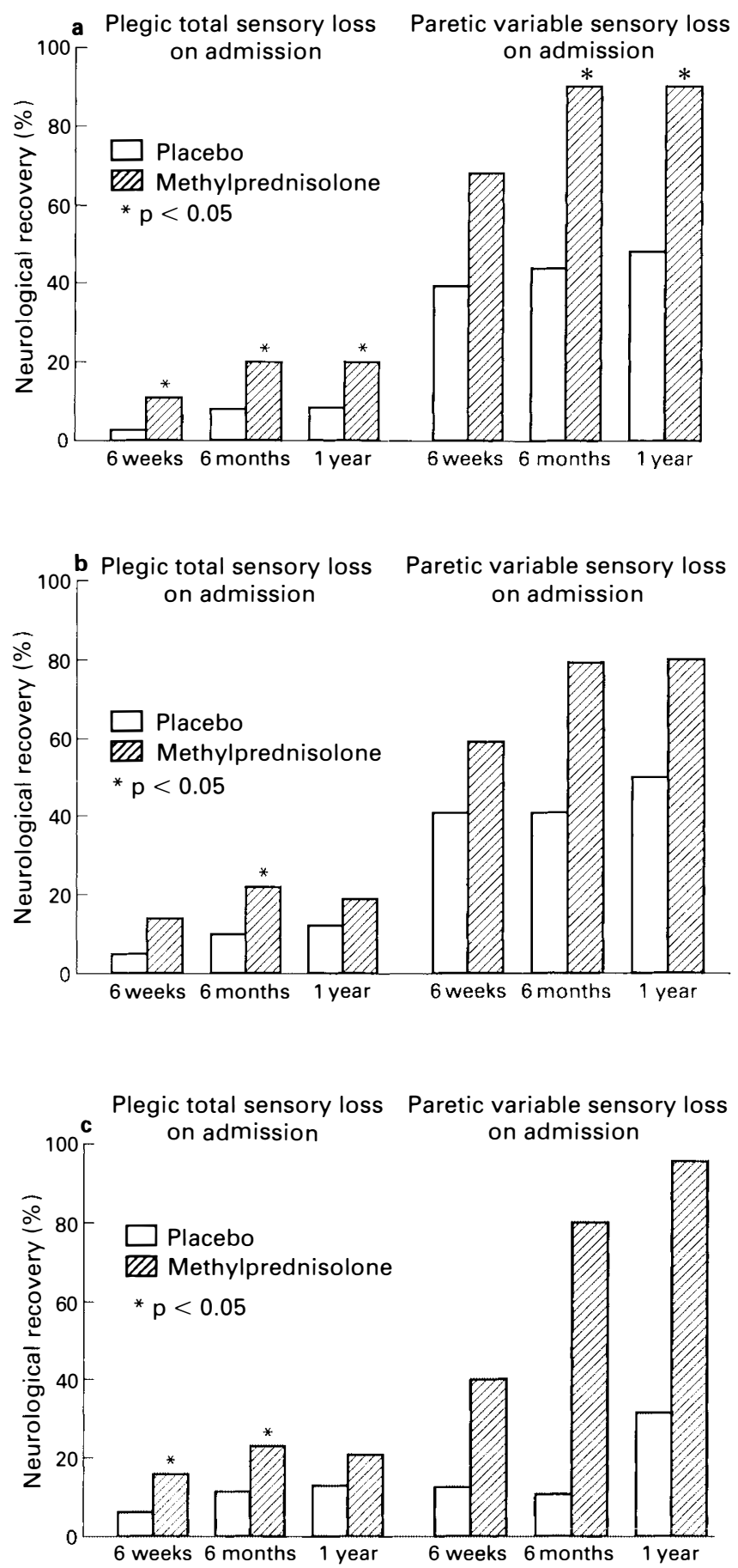

Figure 2 Percent recovery of (a) motor function, (b) pinprick and (c) light touch sensation 6 weeks, 6 months and one year after injury in patients treated with placebo or methylprednisolone within 8 hours of injury, grouped by whether the patient was plegic with total sensory loss or paretic with variable sensory loss in the emergency room. $p$-values refer to analysis of variance ${ }^{7,8}$. 
receiving placebo. ${ }^{8} \mathrm{~A}$ number of factors which may explain why delayed administration of methylprednisolone could inhibit neurological recovery have been reviewed by Hall. ${ }^{9}$

\section{Future prospects for pharmacological therapy}

The NASCIS 2 results have provided, in normal clinical settings, the first support for the hypothesis that a pharmacological agent can reduce the permanent neurological disability that usually follows an acute spinal cord injury. In so doing, the study has validated over 20 years of experimental investigation of the same general hypothesis. The precise mechanisms by which neurological preservation occurs have been widely discussed ${ }^{2,10}$ but remain uncertain. It is likely that several beneficial effects occur.

The NASCIS studies also point to the necessity of evaluating pharmacological therapy under the rigorous conditions of randomized controlled trials. ${ }^{11}$ Not only do therapeutic regimens harbor the potential for serious side effects but the modest degree of recovery achieved is only likely to be reliably observed in relatively large studies. Spinal cord injury is a very heterogeneous condition and the high degree of variability exhibited in the characteristics of the injury, and recovery from it, demand large samples of patients to document valid pharmacological effects.

The NASCIS group of investigators are presently conducting a third trial in which the lipid peroxidation inhibition hypothesis is being explicitly tested. Lipid peroxidation almost certainly continues in the spinal cord for a period longer than 24 hours; thus, it is theoretically possible that by extending the 24-hour NASCIS protocol to 48 hours, greater neurological recovery will be achieved. We are also studying the therapeutic effects of Tirilazad, a drug with especially potent lipid peroxidation inhibition properties, ${ }^{12}$ also administered for 48 hours. Tirilazad lacks any corticosteroidal properties; thus, any complications which the 48-hour regimen of methylprednisolone may incur are unlikely to be seen with Tirilazad. The specific treatments under study in NASCIS 3 are: (1) MPSS $30 \mathrm{mg} / \mathrm{kg}$
15 minute bolus followed by 24 hour MPSS at $5.4 \mathrm{mg} / \mathrm{kg} / \mathrm{hr}$; (2) MPSS $30 \mathrm{mg} / \mathrm{kg} 15$ bolus followed by 48 hour MPSS at $5.4 \mathrm{mg} / \mathrm{kg} / \mathrm{hr}$; and (3) MPSS $30 \mathrm{mg} / \mathrm{kg} 15$ bolus followed by 48 hour Tirilazad at $2.5 \mathrm{mg} / \mathrm{kg}$ every 6 hours.

In a recent report, 16 patients who received GM-1 ganglioside were compared to 18 patients receiving placebo. ${ }^{13}$ This was a Phase 1 randomized trial designed to test GM-1 for safety. However, improved neurological function was observed in the GM-1 patients on both their Frankel grades and motor scores one year after injury. This small study provides strong support for conducting a Phase 3 clinical trial of the efficacy of GM-1 but cannot itself be used as evidence for the routine use of GM-1 in clinical practice. It is of particular interest that unlike NASCIS 2, where methylprednisolone treatment had to start within 8 hours to be efficacious and continued for only 24 hours, GM-1 was started within 48 hours (range 19-72) and was administered for an average of 26 days (range 18-32). Should GM-1 be found to be effective in a larger trial, it will suggest alternative biological mechanisms of recovery, and the possibility of using different pharmacological therapies for treatment both in the early phase of injury and during the later management of the patient.

When Paraplegia was founded 30 years ago, there was no prospect of reducing or reversing paralysis resulting from acute spinal cord injury. In the intervening years, we have seen the evolution of the animal model, numerous experimental studies of pharmacological agents, creation of multicenter randomized trials to evaluate drug therapy, demonstration of the efficacy and safety of one therapy-high dose methylprednisolone-in a human trial, and the promise of additional therapies presently being studied.

\section{Acknowledgement}

This study was supported by grant NS 15078 from the National Institute of Neurological Diseases and Stroke, USA. The study drugs and placebos were provided by the Upjohn Corporation (methylprednisolone) and the Dupont Corporation (naxolone). 


\section{References}

1 Demopoulos HG, Milvy P, Kakari S, Ransohoff J (1972). Molecular aspects of membrane structure in cerebral edema. In: Reulen HJ, Schurmann K, eds. Steroids and Brain Edema. Springer-Verlag, New York.

2 Hall ED (1992). The neuroprotective pharmacology of methylprednisolone: a review. J Neurosurg 76: 13-22.

3 Bracken MB, Collins WF, Freeman, DF et al (1984). Efficacy of methylprednisolone in acute spinal cord injury. JAMA 251: 45-52.

4 Bracken MB, Shepard MJ, Hellenbrand KG, et al (1985). Methylprednisolone and neurologic function one year after injury: results of the national acute spinal cord injury study. J Neurosurg 63: 704-713.

5 Hall ED, Braughler JM (1982). Glucocorticoid mechanisms in acute spinal cord injury: a review and therapeutic rationale. Surg Neurol 18: 320-327.

6 Faden AI, Jacobs TP, Holaday JW (1981). Opiate antagonist improves neurologic recovery after spinal cord injury. Science 211: 493-494.

7 Bracken MB, Shepard MJ, Collins WF, et al (1990). A randomized, controlled trial of methylprednisolone or naloxone in the treatment of acute spinal cord injury: results of the second national acute spinal cord injury study. $N$ Engl J Med 322: 1405-1411.

8 Bracken MB, Shepard MJ, Collins WF, et al (1992). Methylprednisolone or naloxone in the treatment of acute spinal cord injury: one year follow-up results of the second national acute spinal cord injury study. $J$ Neurosurg 76: 23-31.

9 Hall ED (1990) Steroids and neuronal destruction or stabilization. In: Simmonds H, ed. Steroids and Neuronal Activity. CIBA Foundation Symposium No. 153. Wiley and Sons, Chichester.

10 Hsu CY, Dimitrijevic MR (1990). Methylprednisolone in spinal cord injury: the possible mechanism of action. J Neurotrauma 7: 115-119.

11 Bracken MB, Collins WF (1985) Randomized clinical trials of spinal cord injury treatment. In: Becker DP, Povlishock JT, eds. Central Nervous System Trauma Status Report, 1985. National Institute of Neurological and Communicative Disorders and Stroke, Washington, DC.

12 Hall ED, McCall JM, Chase RL, Yonkers PA, Braughler JM (1987). A non-glucocorticoid steroid analog of methylprednisolone duplicates its high-dose pharmacology in models of central nervous system trauma and neuronal membrane damage. J Pharmacol Exp 242: 137-142.

13 Geisler F, Dorsey FC, Coleman WP (1991). Recovery of motor function after spinal cord injurya randomized, placebo-controlled trial with GM-1 ganglioside. N Engl J Med 324: 1829-1838. 